\title{
Etiology of Ocular Infections and Minimum Inhibitory Concentration of Multidrug- Resistant Staphylococcus aureus Isolates to Vancomycin, Ciprofloxacin and Chloramphenicol
}

\section{Samina Thapa ${ }^{1}$, Nabaraj Adhikari ${ }^{2}$, Binod Dhungel ${ }^{2}$, Madhu Thapa ${ }^{3}$, Upendra Thapa Shrestha ${ }^{2}$, Megha Raj Banjara ${ }^{2}$, Komal Raj Rijal' ${ }^{2 *}$ and Prakash Ghimire ${ }^{2}$}

${ }^{1}$ Kantipur College of Medical Sciences, Sitapaila, Kathmandu, Nepal

${ }^{2}$ Central Department of Microbiology, Tribhuvan University, Kirtipur, Nepal

${ }^{3}$ BP Koirala Lions Center for Ophthalmic Studies, Institute of Medicine, Tribhuvan

University, Kirtipur, Nepal

*Corresponding Author: Komal Raj Rijal, Associate Professor, Central Department of

Microbiology, Tribhuvan University, Kirtipur, Kathmandu, Nepal.

\author{
Received: April 05, 2020 \\ Published: May 28, 2020 \\ (C) All rights are reserved by Komal Raj Rijal., \\ et al.
}

\section{Abstract}

Introduction: Ocular infections constitute a significant portion of curable eye diseases. However, in developing countries like Nepal, bacterial and fungal spectrum is not well established for ocular infections. Therefore, ocular infections still remain as the major cause of blindness. This study was sought determine the bacterial and fungal etiology of various ocular infections and assess the antibiotic susceptibility pattern of the bacterial pathogens.

Methods: This cross-sectional study was conducted at BP Koirala Lions Center for Ophthalmic Studies, Kathmandu, Nepal from June 2012 through April 2013. Various ophthalmic specimens of clinical values such as the corneal scraps, conjunctival swab and eye pus and biopsy materials were collected aseptically and processed for culture. After sufficient incubation, isolates were identified by colony morphology, Gram staining and relevant biochemical tests. Identified bacteria; isolates were then subjected under Antibiotic Susceptibility test (AST) by modified Kirby-Bauer disc diffusion method to determine Multi Drug Resistant (MDR) isolates and Minimum Inhibitory Concentration (MIC).

Results: A total number of 810 ocular specimens, mainly from the case of conjunctivitis (644/810) were processed. Of which, $31.97 \%$ (270/810) showed growth on culture medium: 267 (98.85\%) were bacterial, 2 (0.77\%) were fungal (Penicillium spp and Fusarium spp) and $1(0.38 \%)$ was yeast (Rhodotorula) isolate. Gram positives alone accounted for $96.25 \%(257 / 267)$ among bacterial isolates. Staphylococcus aureus (52.96\%) and Escherichia coli (1.48\%) were the most common Gram positive and Gram-negative isolates respectively. Gram positive isolates showed resistance to ciprofloxacin (39.29\%), cephalexin (38.13\%) and amikacin (31.52\%) while gram negatives were resistant to cefazolin (80\%). All isolates of E. coli, Hemophilus influenzae and Serratia spp together with $48.95 \%$ of $S$. aureus were MDR. Among 5 methicillin resistant S. aureus (MRSA) isolates, 2 isolates were vancomycin intermediate $S$. aureus (VISA). Further, the minimum inhibitory concentration (MIC) break points for S. aureus to chloramphenicol, ciprofloxacin and vancomycin were also determined.

Conclusion: Conjunctivitis among adults was the most prevalent case found so far, yielding higher culture positivity. Along with MDR bacterial agents, fungal agents were observed to be posing significant threat in the disease management. Early diagnosis, proper management and empirical treatment are advised to prevent infections related blindness.

Keywords: Ocular Infections; Conjunctivitis; AST; MDR; MRSA; VISA; MIC

\section{Introduction}

There are various ocular and periocular microbial infections including cellulitis, blepharitis, chalazion, conjunctivitis, keratitis, uveitis, endophthalmitis, panophthalmitis, episcleritis, scleritis, pterygium, dacryocystitis, internal and external hordeolum, canaliculitis [1]. Ocular redness, swelling, tearing or discharge, photophobia, ocular pain or discomfort, or foreign body sensation are most common symptoms observed during ocular infections [2]. S. aureus, S. pneumoniae, Bacillus cereus, Coagulase-negative Staphylococci (CONS), Neisseria gonorrhoeae, N. meningitidis, Haemophilus influenzae, Moraxella catarrhalis, Pseudomonas spp, Enteric Gram-negative bacilli, Corynebacterium spp, alpha-haemolytic 
Etiology of Ocular Infections and Minimum Inhibitory Concentration of Multidrug- Resistant Staphylococcus aureus Isolates to Vancomycin, Ciprofloxacin and Chloramphenicol

Streptococci, Micrococcus spp, Beta-haemolytic Streptococci, Corynebacterium diphtheriae are the common bacterial agents for eye infections like conjunctivitis, keratitis and endophthalmitis [1,3]. Fungal infections are caused as opportunistic infections. Fusarium spp, Aspergillus spp, Penicillium spp, Scedosporium spp, Paecilomyces spp, Acremonium spp, Bipolaris spp and yeasts like Candida spp, Cryptococcus spp, Rhodotorula spp, are the major agents to bring the infection [4].

Various socio-demographic factors like age, marital status, income also determine the rate of infection [5]. Globally about 202 million people are visually impaired. The global prevalence of blindness is $0.7 \%$ ranging from $0.3 \%$ in the developed countries to $1.4 \%$ in sub-Saharan Africa [6].

Corneal scarring, tradition remedies, civil war, insufficient medical care, and Vitamin A deficiency (VAD) among children and pregnant women remain as the leading causes of blindness in $\mathrm{Ne}$ pal. Some eye infections are the result of occupational hazard basically observed among agricultural workers of India and Nepal, often caused by organic matter such as rice paddy stalks, dust, grass, wood and animal products [7]. Dirty water, mud and even cow dung used as part of traditional remedies further worsen the case which could otherwise be treated up to $80 \%$ of the cases, if diagnosed promptly [8].

The etiology of ocular infections varies considerably between and within countries [9]. Due to geographical diversity with a known climatic variation, several types of diseases more frequently occur in Nepal [10]. While the poor health facilities, illiteracy, belief on traditional treatments delaying the clinical diagnosis and treatments are the major causes of blindness [11]. Wide range of organisms produce a similar clinical picture, as a result clinical diagnosis does not give an unequivocal indication of the causative organisms $[12,13]$. The initial antimicrobial therapy is often guided by subjective interpretation of clinical features presented [14].

Ocular pathogens are developing resistance towards antibiotics in higher rates that may have serious consequences such as keratitis, endophthalmitis, orbital cellulitis, or panophthalmitis [15]. Nowadays resistance of bacteria to antimicrobial agents has become a worldwide concern. In a study, significant portion of $S$. pneumoniae and $S$. aureus had shown resistance to macrolide antibiotic agents, penicillin, and older fluoroquinolones. However, new generation of fluoroquinolones including third generation levofloxacin and fourth generation moxifloxacin and gatifloxacin have been introduced to counter this problem [16]. In infections like conjunctivitis and corneal infection, topical drops are preferred while Intravitreal antibiotics and possibly subconjunctival and parenteral antibiotics are preferred for endophthalmitis and infection of deep adnexal structures [17]. Among 85\% of methicillin-resistant $S$. aureus (MRSA) isolates were resistant to topical fluroquinolones first-line treatment of ocular infections-- includes the newer 8-methoxy fluoroquinolones, gatifloxacin and moxifloxacin [15]. Similarly, some strains of $H$. influenzae were resistant to chloramphenicol and ampicillin [18].

Emergence of antibiotic resistant strains like MRSA, methicillin resistant $S$. epidermidis (MRSE), vancomycin resistant Staphylococcus aureus (VRSA) and multidrug resistant (MDR) strains of Pseudomonas spp, Haemophilus spp, Streptococcus spp are the major problem in hospital environment. They are associated with increased duration of hospital stay, disseminated infection, increased socioeconomic cost and furthering adverse complications in untreated cases. The study was conducted aiming to identify etiological agent of specific ocular infections and to know the changing resistance pattern of those isolates. Further, the study was conducted to determine the prevalence of MRSA and to evaluate the MIC values of $S$. aureus to vancomycin, ciprofloxacin and chloramphenicol The study thus helps in proper diagnosis and empirical therapy of ocular infections in all Hospital settings.

\section{Materials and Methods}

Study period, site and population

This hospital-based cross-section al study was carried out at B.P. Koirala Lions Centre for Ophthalmic Studies (BPKLCOS), Institute of Medicine, Kathmandu, Nepal from June 2012 through April 2013. Study was conducted within the population defined by physician as the probable case of ocular infections (both bacterial and fungal eye infections) and showing specific clinical symptoms and visiting the respected tertiary eye care center. Patients undergoing ocular surgery and patients on long term treatment for systemic diseases (on steroids, immunosuppressive or anti-cancer drugs) were not included in the study.

A total of 810 different ocular samples were collected from the patients suspected of ocular infections. The personal and demographic information such as personal details, clinical history, clinical symptoms, were obtained by using requisition file of hospital and a structured questionnaire. The laboratory analysis of collected samples was done in pathology laboratory of BPKLCOS and KCMS. All the ocular samples transferred to the laboratory were processed during the study period. 
Etiology of Ocular Infections and Minimum Inhibitory Concentration of Multidrug- Resistant Staphylococcus aureus Isolates to Vancomycin, Ciprofloxacin and Chloramphenicol

\section{Laboratory processing}

Sample collection

Eye specimens such as eye swabs, corneal scrapings, aspirate of vitreous and aqueous fluids, corneal button, were collected by trained physician [19]. The conjunctival surface samples were taken with sterile cotton or calcium alginate cotton swab. For the corneal samples, a sterile Kimura spatula was used and additionally tweezers for sampling tissue remains in the compromised area. Intraocular liquid was sampled with vitreal puncture (vitreous humor) or with paracentesis (aqueous humor), in a syringe under aseptic conditions and sent to the laboratory analysis immediately.

\section{Sample storage and transportation}

The samples collected were immediately inoculated into culture media and also smears were prepared on slides for direct microscopy. Since, the quantity of eye specimen like aqueous (AC) and Vitreous (VIT) were scanty, they were processed for culture immediately. In case of delay, all the specimens were stored at $4^{\circ} \mathrm{C}$ until further lab analysis [20].

\section{Microscopic examination}

The smears prepared on the bedside were examined by following standard Gram's stain technique for bacteria [21] and adhesive tape technique using lactophenol cotton blue (LPCB) and 10\% potassium hydroxide $(\mathrm{KOH})$ analysis for the fungi identification and then observed under microscope [20].

\section{Culture of specimen}

The samples obtained were inoculated into primary isolation media; Blood agar (BA) and Chocolate agar (CA) for isolation of bacteria and into Sabouraud's Dextrose Agar (SDA) plate for isolation of fungi. The chocolate agar plate was always inoculated first. For conjunctival swabs, the swabs of both eyes were inoculated on single media plate using the opposite half of the plate(s). In case of corneal samples, the samples were inoculated on CA and BA plates by $C$ streaking. Whereas for Vitreous (VIT) and Aqueous chamber (AC) culture, the AC or VIT drop were directly inoculated on CA and BA plates by $\mathrm{C}$-streaking. A C-shaped streak was made on culture plates to ensure that growth was from the specimen and not laboratory contaminants. Thioglycolate broth media was used to enrich the both aerobic and anaerobic microorganisms. In case of scant amount of specimen of intraocular fluid submitted in syringe, broth was used to wash out the syringe by drawing up small amount of broth.

The cultures on $\mathrm{BA}$ and $\mathrm{CA}$ were incubated at $37^{\circ} \mathrm{C}$ in $5-7 \% \mathrm{CO}_{2}$ for 48 hrs. The broth media were observed for the turbidity. The cultures were checked at 24 and 48 , reporting the microbial growth in each culture. In the absence of growth, the bacterial culture media were discarded at day 5 .

Similarly, the specimens inoculated for fungal culture on SDA plates were incubated at $25-30^{\circ} \mathrm{C}$. The cultures were checked for fungal growth after 48 hours upto 4 weeks (minimum of 1 week) $[20,21]$.

\section{Isolation and identification of bacteria}

After 24 hours of incubation, the identification procedure was followed. The standard microbiological technique which involved colony morphology, staining reaction, biochemical properties were followed for the identification of organisms [19,21].

\section{Isolation and identification of fungi}

After one week of incubation, the colony characteristics were studied and identification was done by following Standard mycological procedures [22]. Fungal hyphae and spores were observed microscopically by using adhesive tape technique [23]. Indigenous and rare organisms, if present, were reported as "Possible Contamination".

\section{Antimicrobial susceptibility test}

The antimicrobial sensitivity tests of the identified bacteria were performed on commercially available Muller Hinton Agar (MHA) of Hi-Media Company of India. The sensitivity test for $S$. pneumoniae and viridians was performed on MHA with $5 \%$ blood Bacterial susceptibility test to antimicrobial agent was performed in vitro by modified Kirby-Bauer disc diffusion method using fresh broth culture of the isolates. The antibiotics used in the study included amikacin, chloramphenicol, ciprofloxacin, ceftriaxone, ceftazidime, gentamycin, vancomycin, ofloxacin, cefazoline, and the results were interpreted as sensitive, intermediate or resistant by comparing the zone of inhibition as per the guidelines of Clinical and Laboratory Standard Institute [24].

\section{Screening of MRSA}

Further screening of MRSA was conducted among multidrug resistant Staphylococcus aureus. Staphylococcus aureus strains which showed resistant to oxacillin $(1 \mu \mathrm{g})$ at $35^{\circ} \mathrm{C}$ were subjected to test cefoxitin $(30 \mu \mathrm{g})$ to confirm MRSA. In this study if isolates were resistant to at least three classes of antimicrobial agents, then it was regarded as MDR $[25,26]$. MRSA isolates along with other MDR isolates in pure culture were preserved in $20 \%$ glycerol containing tryptic soya broth at $-70^{\circ} \mathrm{C}$ until further investigation. 
Etiology of Ocular Infections and Minimum Inhibitory Concentration of Multidrug- Resistant Staphylococcus aureus Isolates to Vancomycin, Ciprofloxacin and Chloramphenicol

Determination of minimum inhibitory concentration (MIC)

MIC to vancomycin in isolated MRSA and MIC to chloramphenicol and ciprofloxacin in MDR Staphylococcus aureus (ciprofloxacin and chloramphenicol resistant S. aureus) was performed by Agar dilution method following CLSI guidelines [24].

Quality control

Each antibiotics disc and Mueller Hinton agar were checked for each lot number, manufacture date and expiry date and tested for their performance quality with S. aureus ATCC 29213 and E. coli ATCC 25922. Quality of sensitivity tests was maintained by maintaining the thickness of Mueller-Hinton agar at $4 \mathrm{~mm}$ and the $\mathrm{pH}$ at $7.2-7.4$

Data management and analysis

The data were filled in data entry sheet and were analyzed using Statistical Package for Social Science (SPSS) software (Version 16.0) and Microsoft EXCEL.

\section{Results}

A total of 810 ocular samples were processed for culture and all culture positive samples were further processed for antibiotic susceptibility test.

Five different types of samples were collected from the suspected patients with more than twenty different infections. Highest number of samples were from the clinical cases of conjunctivitis; the sample being conjunctival swabs ( $n=767 ; 94.69 \%)$. Here, the $p$ value $(p<0.05)$ showed significant relationship between type of sample and culture positivity (Table 1 ).

Age and sex wise distribution for isolated organisms

Among the different age group of patients, highest number of infections were found in age group 19 - 60 years that included 325 (40.12\%); male patient: 169 and female patient: 156 . The relationship between age and gender with culture positivity was observed to be insignificant $(\mathrm{P}>0.05)$ (Table 2).
Culture positivity according to the clinical diagnosis of infections

More than 20 different types of clinical ocular cases were included in this study. The case of acute bacterial conjunctivitis was the highest having 79.5\% $(\mathrm{n}=644)$ though its culture positivity was only $30.12 \%$. The $100 \%$ culture positivity was observed in case of Impetigo and Blepharospasm Conjunctivitis though the cases reported on this disease were only 1 and 2 respectively. These cases of culture positive were followed by dacryocystitis $75 \%$ (n = 6) (Table 3).

Generalized pattern of organisms isolated from the samples

Out of the 270 different organisms isolated maximum number of organisms belong to Gram positive bacteria i.e. 239 (88.52\%) (Figure 1).

Bacterial and fungal isolates identified from the samples of ocular infections

Among gram positive isolates $S$. aureus was the dominant organisms isolated; 137 out of 267 (51.31\%) where as in case of Gram-negative isolates E. coli was frequently isolated; 4 out of 267 (1.48\%). Among fungal isolates only 2 genus, Penicillium spp and Fusarium spp and only one genus of yeast Rhodotorula spp was isolated (Table 4).

Antibiotic susceptibility pattern observed in Streptococci isolates

Among Gram positive isolates from the ocular infections S. pneumoniae was found to be highly resistant to Amikacin (72.73\%). Group D Streptococci were found to be highly resistant to Tobramycin (43.75\%), viridans streptococci were highly resistant to Amikacin and Tobramycin (50\%) and Streptococcus pyogenes were highly resistant to Tobramycin, Gentamycin, Chloramphenicol and Cephalexin (33.33\%). Bacillus spp, Micrococcus spp and Corynebacterium spp were found to be susceptible to all the antibiotics used (Table 5).

\begin{tabular}{|l|c|c|c|c|c|c|}
\hline \multicolumn{1}{|c|}{ Sample Type } & $\begin{array}{c}\text { Total } \\
\text { Number }\end{array}$ & $\begin{array}{c}\text { Culture positive for } \\
\text { bacteria (\%) }\end{array}$ & $\begin{array}{c}\text { Culture positive } \\
\text { for fungi (\%) }\end{array}$ & $\begin{array}{c}\text { Culture positive } \\
\text { for yeast (\%) }\end{array}$ & $\begin{array}{c}\text { Total culture } \\
\text { positive (\%) }\end{array}$ & $\begin{array}{c}\text { P } \\
\text { value }\end{array}$ \\
\hline Conjunctival swab & 767 & $235(30.64)$ & $0(0.00)$ & $0(0.00)$ & $235(30.64)$ & 0.003 \\
\hline Corneal scraps & 33 & $15(45.45)$ & $2(6.06)$ & $1(3.03)$ & $0(54.55)$ \\
\hline $\begin{array}{l}\text { Vitreous and Aqueous } \\
\text { Taps }\end{array}$ & 8 & $4(50)$ & $0(0.00)$ & $4(50)$ \\
\hline Eye Pus & 2 & $2(100)$ & $0(0.00)$ & $0(0.00)$ & $2(100)$ \\
\hline Total & 810 & $256(31.60)$ & $2(0.25)$ & $1(0.12)$ & $259(31.98)$ & \\
\hline
\end{tabular}

Table 1: Distribution of sample collected according to its type. 
Etiology of Ocular Infections and Minimum Inhibitory Concentration of Multidrug- Resistant Staphylococcus aureus Isolates to Vancomycin, Ciprofloxacin and Chloramphenicol

\begin{tabular}{|c|c|c|c|c|c|c|c|}
\hline $\begin{array}{l}\text { Age group } \\
\text { (year) }\end{array}$ & Male & Culture Positives (\%) & Female & $\begin{array}{c}\text { Culture positives } \\
(\%)\end{array}$ & $\begin{array}{c}\text { Total } \\
\text { Sample }\end{array}$ & $\begin{array}{l}\text { Total Culture } \\
\text { Positive (\%) }\end{array}$ & p-value \\
\hline$<1$ & 89 & 42 (47.19) & 35 & $21(60.00)$ & 124 & $63(50.81)$ & \\
\hline $1-5$ & 92 & $28(30.43)$ & 48 & $16(33.33)$ & 140 & $44(31.43)$ & \\
\hline $6-18$ & 100 & $30(30.00)$ & 72 & $18(25.00)$ & 172 & 48 (27.91) & 0.354 \\
\hline $19-60$ & 169 & $43(25.44)$ & 156 & 41 (26.28) & 325 & $84(25.85)$ & \\
\hline$>60$ & 28 & 11 (39.29) & 21 & $9(42.86)$ & 49 & $20(40.82)$ & \\
\hline Total & 478 & $154(32.22)$ & 332 & 105 (31.62) & 810 & 259 (31.98) & \\
\hline
\end{tabular}

Table 2: Age and sex wise distribution of patients according to culture positivity.

Note: $<1=$ Infants and neonates, $1-5=$ Toddlers, $6-18=$ Children, $19-60=$ Adults and $>60=$ Seniors.

\begin{tabular}{|c|c|c|c|c|c|}
\hline Clinical Diagnosis & $\begin{array}{c}\text { Total } \\
\text { number }\end{array}$ & $\begin{array}{c}\text { Culture Positive } \\
\text { for Bacteria }\end{array}$ & $\begin{array}{l}\text { Culture Positive } \\
\text { for Fungi }\end{array}$ & $\begin{array}{c}\text { Culture Positive } \\
\text { for yeast }\end{array}$ & $\begin{array}{c}\text { Total Culture Positive } \\
(\%)\end{array}$ \\
\hline Acute Bacterial conjunctivitis & 644 & 194 & 0 & 0 & $194(30.12)$ \\
\hline Endophthalmitis & 9 & 4 & 0 & 0 & $4(44.44)$ \\
\hline Corneal ulcer & 21 & 11 & 2 & 0 & $13(61.90)$ \\
\hline Blepharoconjunctivitis & 14 & 3 & 0 & 0 & $3(21.42)$ \\
\hline CNLDO & 35 & 11 & 0 & 0 & $11(31.43)$ \\
\hline $\begin{array}{l}\text { Pseudomembranous acute bacterial } \\
\text { conjunctivitis }\end{array}$ & 16 & 4 & 0 & 0 & $4(25)$ \\
\hline Preseptal cellulitis & 8 & 3 & 0 & 0 & $3(37.50)$ \\
\hline Chalazion & 4 & 0 & 0 & 0 & $0(0)$ \\
\hline Viral keratoconjunctivitis & 8 & 3 & 0 & 0 & $3(37.5)$ \\
\hline Blepharospasm Conjunctivitis & 2 & 2 & 0 & 0 & $2(100)$ \\
\hline Follicular conjunctivitis & 5 & 0 & 0 & 0 & $0(0)$ \\
\hline Phlyctenular conjunctivitis & 6 & 0 & 0 & 0 & $0(0)$ \\
\hline Dacryocystitis & 8 & 6 & 0 & 0 & $6(75)$ \\
\hline Stye & 8 & 4 & 0 & 0 & $4(50)$ \\
\hline Keratitis & 5 & 1 & 0 & 1 & $2(40)$ \\
\hline Hordeolum & 3 & 2 & 0 & 0 & $2(66.67)$ \\
\hline Impetigo & 1 & 1 & 0 & 0 & $1(100)$ \\
\hline Trauma & 6 & 3 & 0 & 0 & $3(50)$ \\
\hline Others & 7 & 4 & 0 & 0 & $4(57.14)$ \\
\hline Total & 810 & 256 & 2 & 1 & 259 (31.98) \\
\hline
\end{tabular}

Table 3: Culture positivity according to the clinical diagnosis of infections. 
Etiology of Ocular Infections and Minimum Inhibitory Concentration of Multidrug- Resistant Staphylococcus aureus Isolates to Vancomycin, Ciprofloxacin and Chloramphenicol

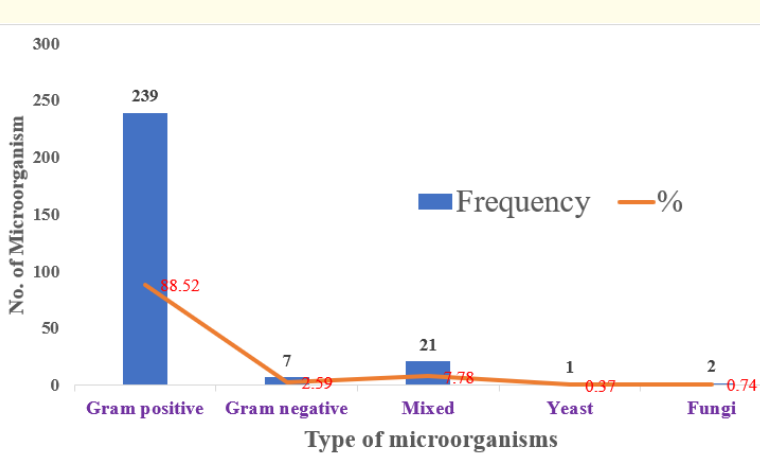

Figure 1: Growth pattern of microorganism from suspected eye infection patients.
Antibiotic susceptibility pattern observed in Staphylococci and Enterococci isolates

S. aureus is among the highly isolated Gram-positive bacteria $52.96 \%$ of total bacterial isolates. They were found to be highly resistant to ciprofloxacin (52.45\%). Among 143 S. aureus, $3.4 \%$ (5/143) of them were found to be MRSA. Eighty percent of these MRSA isolates were found to be resistant to all cephalosporins namely: cefazolin, cephalexin and cefotaxime, amoxycillin and ciprofloxacin. Imipenem and azithromycin were found to be $100 \%$ effective on MRSA isolates. CONS were found to be $48 \%$ resistant to cephalexin while Enterococcus spp were found to be $57.14 \%$ resistant to ciprofloxacin and cephalexin (Table 6).

\begin{tabular}{|c|c|c|c|}
\hline Isolates & Pure Isolates & Mixed with other bacteria & Total number (\%) \\
\hline \multicolumn{4}{|l|}{ Gram Positive } \\
\hline Staphylococcus aureus & 137 & 6 & $143(52.96)$ \\
\hline Streptococcus pneumoniae & 52 & 3 & $55(20.37)$ \\
\hline CONS & 23 & 2 & $25(9.26)$ \\
\hline Group D Streptococci & 13 & 3 & $16(5.93)$ \\
\hline Enterococcus spp & 6 & 1 & $7(2.59)$ \\
\hline Viridan Streptococci & 3 & 1 & $4(1.48)$ \\
\hline Streptococcus pyogene & 3 & - & $3(1.11)$ \\
\hline Bacillus spp & 1 & 1 & $2(0.74)$ \\
\hline Corynebacterium diphtheriae & 1 & - & $1(0.37)$ \\
\hline Micrococcus spp & - & 1 & $1(0.37)$ \\
\hline \multicolumn{4}{|l|}{ Gram Negative } \\
\hline Escherichia coli & 2 & 2 & $4(1.48)$ \\
\hline Pseudomonas spp & 3 & - & $3(1.11)$ \\
\hline Haemophilus influenzae & 1 & 1 & $2(0.74)$ \\
\hline Serratia spp & 1 & - & $1(0.37)$ \\
\hline \multicolumn{4}{|l|}{ Fungi } \\
\hline Fusarium spp & 1 & - & $1(0.37)$ \\
\hline Penicillium spp & 1 & - & $1(0.37)$ \\
\hline \multicolumn{4}{|l|}{ Yeast } \\
\hline Rhodotorula spp & 1 & - & $1(0.37)$ \\
\hline Total & 249 & 21 & $270(100)$ \\
\hline
\end{tabular}

Table 4: Bacterial and fungal isolates identified from the samples of ocular infections.

Note: CONS: Coagulase Negative Staphylococci. 
Etiology of Ocular Infections and Minimum Inhibitory Concentration of Multidrug- Resistant Staphylococcus aureus Isolates to Vancomycin, Ciprofloxacin and Chloramphenicol

\begin{tabular}{|l|c|c|c|c|}
\hline \multirow{2}{*}{$\begin{array}{c}\text { Anti- } \\
\text { biotics }\end{array}$} & $\begin{array}{c}\text { Streptococcus } \\
\text { pneumoniae }\end{array}$ & $\begin{array}{c}\text { Group D } \\
\text { Streptococci }\end{array}$ & $\begin{array}{c}\text { Viridan } \\
\text { Streptococci }\end{array}$ & $\begin{array}{c}\text { Streptococcus } \\
\text { pyogenes }\end{array}$ \\
\cline { 2 - 5 } & $\mathbf{n = 5 5}$ & $\mathbf{n = 1 6}$ & $\mathbf{n = 4}$ & $\mathbf{n = 3}$ \\
\hline AK & $40(72.73)$ & $5(31.25)$ & $2(50)$ & $0(0.00)$ \\
\hline TB & $32(58.18)$ & $7(43.75)$ & $2(50)$ & $1(33.33)$ \\
\hline G & $8(14.55)$ & $3(18.75)$ & $1(25)$ & $1(33.33)$ \\
\hline C & $2(3.64)$ & $2(12.5)$ & $0(0.00)$ & $0(0.00)$ \\
\hline CIP & $7(12.73)$ & $6(37.5)$ & $0(0.00)$ & $1(33.33)$ \\
\hline OF & $4(7.27)$ & $4(25)$ & $1(25)$ & $0(0.00)$ \\
\hline GAT & $0(0.00)$ & $0(0.00)$ & $0(0.00)$ & $0(0.00)$ \\
\hline CZ & $5(9.09)$ & $3(18.75)$ & $1(25)$ & $0(0.00)$ \\
\hline CN & $7(12.73)$ & $6(37.5)$ & $0(0.00)$ & $1(33.33)$ \\
\hline CTX & $4(7.27)$ & $2(12.5)$ & $0(0.00)$ & $0(0.00)$ \\
\hline VA & $0(0.00)$ & $1(6.25)$ & $0(0.00)$ & $0(0.00)$ \\
\hline COT & $0(0.00)$ & $0(0.00)$ & $0(0.00)$ & $0(0.00)$ \\
\hline
\end{tabular}

Table 5: Antibiotic susceptibility pattern observed in Streptococci isolates.

\begin{tabular}{|l|c|c|c|c|}
\hline \multirow{2}{*}{$\begin{array}{c}\text { Antibiotics } \\
\text { used }\end{array}$} & CONS & $\begin{array}{c}\text { Enterococcus } \\
\text { spp }\end{array}$ & S. aureus & MRSA isolates \\
& $\mathbf{n = 2 5}$ & $\mathbf{n = 7}$ & $\mathbf{n = 1 4 3}$ & $\begin{array}{c}\text { Resistant; n/N } \\
\text { (\%) }\end{array}$ \\
\cline { 2 - 4 } & $\mathbf{R}(\%)$ & $\mathbf{R}(\%)$ & $\mathbf{R}(\%)$ & \\
\hline AK & $4(16)$ & $3(42.86)$ & $27(18.88)$ & $1 / 5(20)$ \\
\hline TB & $3(12)$ & $2(28.57)$ & $29(20.28)$ & $3 / 5(60)$ \\
\hline G & $2(8)$ & $2(28.57)$ & $11(7.69)$ & $2 / 5(40)$ \\
\hline C & $3(12)$ & $1(14.29)$ & $27(18.88)$ & $2 / 5(40)$ \\
\hline CIP & $8(32)$ & $4(57.14)$ & $75(52.45)$ & $4 / 5(80)$ \\
\hline OF & $5(20)$ & $3(42.86)$ & $60(41.96)$ & $3 / 5(60)$ \\
\hline GAT & $0(0.00)$ & $1(14.29)$ & $16(11.19)$ & $1 / 5(20)$ \\
\hline CZ & $5(20)$ & $1(14.29)$ & $35(24.48)$ & $4 / 5(80)$ \\
\hline CN & $12(48)$ & $4(57.14)$ & $68(47.55)$ & $4 / 5(80)$ \\
\hline CTX & $5(20)$ & $1(14.29)$ & $53(37.06)$ & $4 / 5(80)$ \\
\hline VA & $0(0.00)$ & $0(0.00)$ & $5(3.49)$ & $3 / 5(60)$ \\
\hline COT & $1(4)$ & $1(14.29)$ & $32(22.38)$ & $1 / 5(20)$ \\
\hline DO & - & - & $9(6.29)$ & $1 / 5(20)$ \\
\hline AZM & - & - & $16(11.19)$ & $0 / 5(0.00)$ \\
\hline AMX & - & - & $35(24.48)$ & $4 / 5(80)$ \\
\hline IPM & - & - & $0(0.00)$ & $0 / 5(0.00)$ \\
\hline CX & - & - & $5(3.49)$ & $5 / 5(100)$ \\
\hline OX & - & - & $5(3.49)$ & $5 / 5(100)$ \\
\hline
\end{tabular}

Table 6: Antibiotic susceptibility pattern observed in Staphylococci and Enterococci isolates.

Note: Ak: Amikacin; TB: Tobramycin; G: Gentamicin;

C: Chloramphenicol; CIP: Ciprofloxacin; OF: Ofloxacin;

GAT: Gatifloxacin, CZ: Cefazolin; CN: Cephalexin; CTX: Cefotaxime;

VA: Vancomycin; COT: Cotrimoxazole; DO: Doxycycline

Hydrochloride; AZM: Azithromycin; AMX: Amoxycillin;

IPM: Imipenem; CX: Cefoxitin; OX: Oxacillin.

\section{The MDR pattern of bacterial isolates}

Out of 267 bacterial isolates, 37.08\% (n = 99) of isolates were found to be MDR. Among all Gram-positive isolates Staphylococcus aureus was found to have highest percent of MDR strains i.e. $48.95 \%$ of total Staphylococcus aureus isolates which is $70.70 \%$ of total MDR strains in this study. Among Gram negative isolates all isolated strains of E. coli, Serratia spp and Haemophilus influenzae strains were found to be multidrug resistant (Table 7).

MIC of ciprofloxacin on MDR S. aureus isolates

Among 39 S. aureus isolates, 3 isolates were found to grow in 32 $\mu \mathrm{g} / \mathrm{ml}, 2$ isolates grew in $16 \mu \mathrm{g} / \mathrm{ml}, 10$ isolates grew in $8 \mu \mathrm{g} / \mathrm{ml}, 8$ isolates grew on $4 \mu \mathrm{g} / \mathrm{ml}$. 5 isolates were found to be ciprofloxacin intermediate. Remaining 10 isolates grew on $1 \mu \mathrm{g} / \mathrm{ml}$ or less ciprofloxacin concentration (Figure 2).

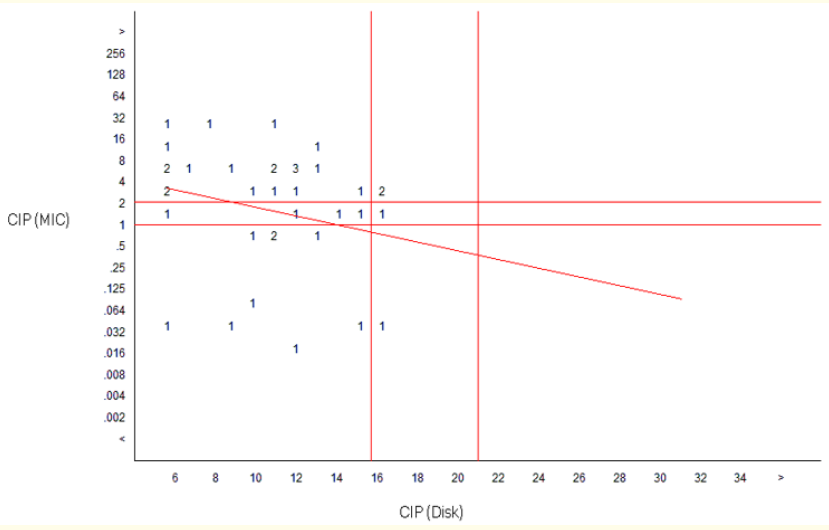

Figure 2: MIC of ciprofloxacin on MDR S. aureus isolates.

MIC of chloramphenicol on MDR S. aureus isolates

Among twelve $S$. aureus isolates, 3 isolates were found growing in $32 \mu \mathrm{g} / \mathrm{ml}, 4$ isolates grew in $4 \mu \mathrm{g} / \mathrm{ml}$ and 5 isolates grew in $2 \mu \mathrm{g} /$ $\mathrm{ml}$. The break point was found to be $13 \mathrm{~mm}$. Three isolates were found to be chloramphenicol resistant (Figure 3).

\section{MIC of vancomycin among MRSA isolates}

Among 5 MRSA isolates, 2 isolates were found to be vancomycin intermediates growing in 4 and $8 \mu \mathrm{g} / \mathrm{ml}$ concentration each. The remaining three isolates grew at $2 \mu \mathrm{g} / \mathrm{ml}$ concentration of vancomycin (Figure 4).

\section{Discussion}

In our study, highest volume (94.69\%) of samples was obtained from the clinical cases of conjunctivitis. This result is in contrast to that reported by other study [27] as the highest sample dealt in this study was corneal scraps showing higher prevalence of corneal 


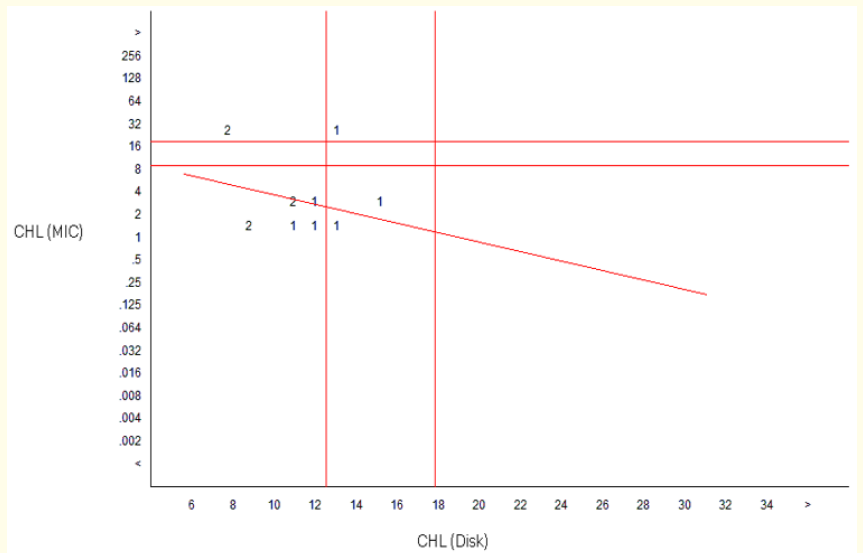

Figure 3: MIC of Chloramphenicol on MDR S. aureus isolates.

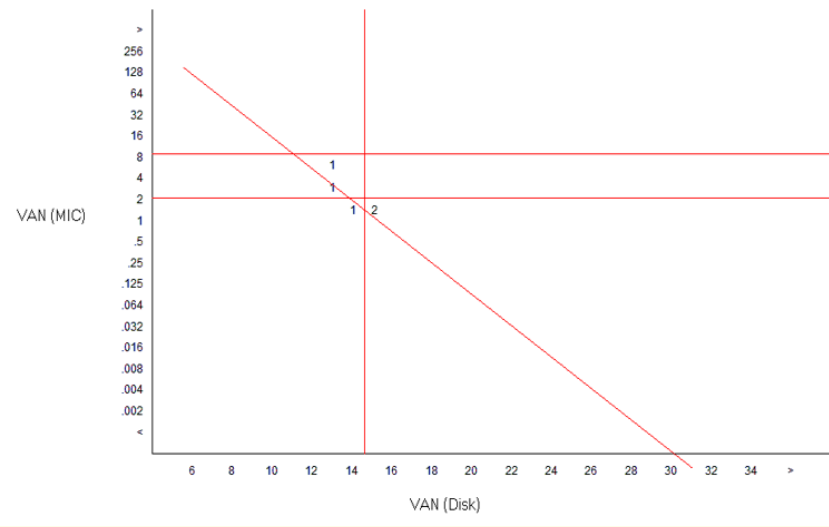

Figure 4: MIC of vancomycin among MRSA isolates.

ulcers. However, in the present study, culture positivity was higher (54.55\%) in corneal scraps beside eye pus that showed absolute positivity. Though corneal scraps are referred as sample for few ocular infections, they were more relevant in giving positive results in this study. It is coupled to the fact that there may be only a few organisms in a corneal ulcer, means that adequate clinical material must be obtained in various culture media. As a result, there is traditional practice of taking multiple scrapes from the ulcer and directly plating the material onto several culture media. Hence there are less chances of sampling error and false negative results [28]. Use of direct plating method and enrichment media like BHI for corneal scraps has led to its high positive rate as BHI provides the excellent recovery of fastidious organisms [29].

Here in this study bacterial isolates were in very high proportion as compared to fungal and yeast isolates of which Gram positives were the most frequently isolated ones. This was similar to

\begin{tabular}{|l|c|c|}
\hline \multirow{2}{*}{ Organism Isolated } & \multicolumn{2}{|c|}{ Drug Resistant Pattern } \\
\cline { 2 - 3 } & Total (\%) & MDR Number (\%) \\
\hline Staphylococcus aureus & $\begin{array}{c}143 \\
(53.56)\end{array}$ & $70(48.95)$ \\
\hline CONS & $25(9.36)$ & $7(28)$ \\
\hline Streptococcus pneumoniae & $55(20.59)$ & $6(10.90)$ \\
\hline Streptococcus pyogenes & $3(1.12)$ & $0(0.00)$ \\
\hline Viridan Streptococci & $4(1.49)$ & $1(25)$ \\
\hline Group D Streptococci & $16(5.99)$ & $3(18.75)$ \\
\hline Enterococcus spp & $7(2.62)$ & $3(42.86)$ \\
\hline Bacillus spp & $2(0.75)$ & $0(0.00)$ \\
\hline Corynebacterium spp & $1(0.37)$ & $0(0.00)$ \\
\hline Micrococcus spp & $1(0.37)$ & $0(0.00)$ \\
\hline Haemophilus influenza & $2(0.75)$ & $2(100)$ \\
\hline Pseudomonas spp & $3(1.12)$ & $2(66.67)$ \\
\hline Escherichia coli & $4(1.49)$ & $4(100)$ \\
\hline Serratia spp & $1(0.37)$ & $1(100)$ \\
\hline Total & $267(100)$ & $99(37.08)$ \\
\hline
\end{tabular}

Table 7: The MDR pattern of bacterial isolates.

Note: CONS: Coagulase Negative Staphylococci

some previous studies [30,31] whereas is in contrast to others [32]. Bacterial isolates are increasingly becoming imperative both clinically and therapeutically as biological agents of ocular infections throughout the world [32]. In the present study, S. aureus was the most frequently isolated bacteria among Gram positive isolates as the highest sample number dealt in this study was conjunctival swab and S. aureus is the leading cause of conjunctivitis and is most common cause of conjunctivitis in adults while children are most prone to $H$. influenzae [33]. In this study, E. coli was the predominant (40\%) among total Gram negatives followed by Pseudomonas spp (30\%), Haemophilus influenzae (20\%) and Serratia spp (10\%). This result was in contrast to different studies which revealed $P$. aeruginosa as the predominant among Gram-negative isolates $[34,35]$.

Antibacterial treatments are cheaper and are readily available and its frequent and widespread use has developed resistance of organisms to many commonly used antibiotics [36]. In this study only $3.49 \%$ were found as MRSA. Studies reported that risk factors for incidence of MDR MRSA are inappropriate self-medication with antibiotics by patients, their perception, compliance, physicians prescribing practices and antibiotic use in hospital [37]. Our study reported nearly half of the VISA isolates (2/5) among all MRSA isolates. This finding can be compared with some reports from India [38] while is not in harmony with some other from the same [39]. 
Etiology of Ocular Infections and Minimum Inhibitory Concentration of Multidrug- Resistant Staphylococcus aureus Isolates to Vancomycin, Ciprofloxacin and Chloramphenicol

This study reported on the increasing trend of the number of MDR bacterial isolates (37.08\%) showing resistance to more than three different classes of antibiotics. All isolates of Haemophilus influenzae and Escherichia coli followed by Pseudomonas spp (66.67\%), Staphylococcus spp (48.95\%), Enterococcus spp (42.86\%), Viridan Streptococci (25\%), Group D Streptococci (18.75\%), and S. pneumoniae (10.90\%). and a single Serratia spp isolated in study was found to be MDR. Though E. coli is rarely found in the normal flora of conjunctiva, they are most commonly encountered as the source of infection in ophthalmic neonatorum [40]. Pseudomonas spp is the frequent cause of Gram-negative infections like keratitis that can affect various ocular sites in immunocompromised patients and are often resistant to multiple antimicrobial agents [41]. Number of MDR strain in ocular infection has increased than reported from a previous study in Nepal [27].

In our study, S. aureus ATCC 29213 exhibited sensitivity to ciprofloxacin, chloramphenicol and vancomycin while was highly resistant $(24 / 143)$ or intermediate $(5 / 143)$ to ciprofloxacin. This finding is in harmony with some previous findings [42,43]. The rapid emergence of ciprofloxacin resistance could be probably due to the indiscriminate and empirical use of these drugs $[43,44]$. Studies showed that fluroquinolone resistance in S. aureus is widespread, which is mainly due to three mechanisms: mutation in nor A gene encoding a membrane- associated active efflux pump, mutation in gyrA and gyrB genes encoding DNA gyrase and mutation in grIA and grIB genes, which encode DNA topoisomerase IV [44].

Similarly, three isolates of $S$. aureus were extremely resistant to chloramphenicol. The finding is similar to previous one [45] and is in contrast to other one [43]. The resistant mechanism on the other hand could be due to the most common mechanism against Beta-lactams i.e. enzymatic inactivation or destruction of the drug molecule, rendering it ineffective at the target site of action [43].

Among 5 MRSA isolates, 2 isolates were found as VISA. Various researchers have reported the prevalence of VISA indicating the potential crisis of effective bactericidal antibiotics against this organism $[46,47]$. Similar findings on VISA were observed from Nepal [48], India [38,39,49]. The report of VRSA from any part of the world has become an international concern as it could well be the next superbug [49]. It is suggested that topical application to the cornea may achieve a very different concentration and bioavailability in the tissue than the serum levels. Thus, MIC is considered as an important measure for evaluating the potential effectiveness of topically applied antimicrobials in the treatment of diseases like bacterial keratitis [50].
This study can be a reference tool to establish the burden of ophthalmic infections in clinical settings. However, broad based surveillance in community settings in Nepal is still lacking which is felt during our literature survey and also seriously affected our study design. Moreover, our study was limited to phenotypic analysis of AMR excluding molecular identification and characterization of wide sorts of genes such as mecA in MRSA, ESBL in Gram negative Enterobacteriaceae isolates. We suggest the molecular characterization in future researches as these analyses are rapid, specific and reliable. This study can also be useful to the concerned authorities to review existing health policies and practices thereby discouraging rampant misuse of antibiotics, self-medication practices, poor quality of medication and diagnostic tools, inadequate doses and lastly poor surveillance.

\section{Conclusion}

Conjunctivitis is the main ocular infection and Gram-positive bacteria were most prevalent than Gram negatives. MDR is yet again identified as the major threat to modern empirical regimens. Of the isolated S. aureus, MRSA and VISA is performed which implied that the vancomycin followed by gatifloxacin and gentamycin could be the most efficient drugs for Gram positive isolates and cotrimoxazole, imipenem and piperacillin/ tazobactam for Gram negatives. However, their ocular toxicity needs to be determined. From this study we can also conclude that fungal infection is among the most sight threatening complication and its early diagnosis and study of their AST pattern is vital in treatment of ocular infections due to fungal etiology. Beside all these results this study emphasizes on proper management of empirical treatment and further management of MDR isolates.

\section{Acknowledgements}

We would like to express our sincere gratitude and admiration to all the patients for their involvement in the study.

\section{Availability of Data and Materials}

All data pertaining to this study are within the manuscript.

\section{Author's Contributions}

Samina Thapa and Nabaraj Adhikari have equally contributed to this study. All the authors made substantial contribution to the study. They read and approved the final manuscript.

\section{Consent for Publication}

Not applicable. 
Etiology of Ocular Infections and Minimum Inhibitory Concentration of Multidrug- Resistant Staphylococcus aureus Isolates to Vancomycin, Ciprofloxacin and Chloramphenicol

\section{Competing Interests}

All the authors declared that they have no competing interests.

\section{Bibliography}

1. Teweldemedhin M., et al. "Bacterial profile of ocular infections: a systematic review". BMC Ophthalmology 17 (2017): 212.

2. Montessori V., et al. "Epidemic kerato-conjunctivitis outbreak at a tertiary referral eye care clinic". American Journal of Infection Control 269.4 (1998): 399-405.

3. Crain EF., et al. "Identifying children at low risk for bacterial conjunctivitis". The Archives of Pediatrics and Adolescent Medicine 164.1 (2010): 263-267.

4. Thomas M and Taylor S. "Antibiotic choices for common infections". Bangalore Political Action Committee (NZ) (2010).

5. Epling John. "Bacterial conjunctivitis". BMJ Clinical Evidence 10.1 (2007): 704-706.

6. Pascolini D and Mariotti SP. "Global estimates of visual impairment: 2010". British Journal of Ophthalmology 96.5 (2012): 614-618.

7. Tewari A., et al. "Epidemiological and microbiological profile of infective keratitis in Ahmedabad". Indian Journal of Ophthalmology 60.4 (2012): 267-272.

8. Amza A., et al. "Community risk factors for ocular Chlamydia infection in Niger: pre-treatment results from a cluster- randomized trachoma trial". PLOS Neglected Tropical Diseases 6.4 (2012): 1586-1593.

9. Leck AK., et al. "Aetiology of suppurative corneal ulcers in Ghana and south India, and epidemiology of fungal keratitis". British Journal of Ophthalmology 86.11 (2002): 1211-1215.

10. Dhakhwa K., et al. "Causative organisms in microbial keratitis, their sensitivity pattern and treatment outcome in western Nepal”. Nepalese Journal of Ophthalmology 4.7 (2012): 119127.

11. Pandey PR. "Study of Ocular Emergencies in Nepal Eye Hospital". PMJNI 9.2 (2009).

12. Florakis GJ., et al. "Scanning slit confocal microscopy of fungal keratitis". Archives of Ophthalmology 115.11 (1997): 14611463.
13. Sridhar MS., et al. "Treatment and outcome of nocardia Keratitis". Cornea 20.5 (2001): 458-462.

14. Wani VP., et al. "Endophthalmitis after virectomy and virectomy combined with phacoemulsification: incidence and visual outcomes". The European Journal of Ophthalmology 19.6 (2009): 1044-1049.

15. McDonald M and Blondeau JM. "Emerging antibiotic resistance in ocular infections and the role of fluoroquinolones". Journal of Cataract and Refractive Surgery 36.1 (2010): 1588-1598.

16. Mather R., et al. "Fourth generation fluoroquinolones: new weapons in the arsenal of ophthalmic antibiotics". American Journal of Ophthalmology 133.4 (2002): 463-466.

17. Synder RW and Glasser DB. "Antibiotic therapy for ocular infection". Westmed Medical 161.1 (1994): 579-584.

18. Campos Jose., et al. "Susceptibility Studies of Multiply Resistant Haemophilus influenza Isolated from Pediatric Patients and Contacts". Antimicrob Agents Chemother 25.1 (1984): 706709.

19. Isenberg HD. "Ocular cultures". In: Clinical Microbiology Procedure Handbook. Hall GS and York MK (editions), $2^{\text {nd }}$ edition, Volume 1, section 3.10. ASM press, Washington DC (2004).

20. Prajna L and Rajlakshmi CP. "Ocular microbiology”. Aravinda Eye hospitals and Postgraduate institute of Ophthalmology, Madurai, India (2005).

21. Cheesebrough M. "District Laboratory Practice in Tropical Countries". Part 2 Cambridge University Press, London (2000): 225-392.

22. Jones DB., et al. "Laboratory diagnosis of ocular infections". Washington DC: American Society for Microbiology (1981).

23. Larone DH. "Medical important fungi, a guide to identification, $3^{\text {rd }}$ Edition". ASM press (2005).

24. Clinical and laboratory standard institute (CLSI). Performance standards for antimicrobial susceptibility testing; TwentyFirst information Supplement". CLSI, M100-S20.CLSI, Wayne PA (2011)

25. Magiorakos AP., et al. "Multidrug-resistant, extensively drugresistant and pandrug-resistant bacteria: an international expert proposal for interim standard definitions for acquired resistance". Clinical Microbiology and Infection 18.1 (2012): 268-281. 
26. Simner PJ., et al. "Prevalence and characterization of extended-spectrum beta-lactamase-and AmpC beta-lactamase-producing Escherichia coli: results of the CANWARD 2007-2009 study". Diagnostic Microbiology and Infectious Disease 69.3 (2011): 326-334.

27. Suwal S., et al. "Microbiological profile of corneal ulcer cases diagnosed in a tertiary care ophthalmological institute in $\mathrm{Ne}$ pal". BMC Ophthalmology 16 (2016): 209.

28. Allan BDS and Dart JKG. "Strategies for the management of microbial keratitis". The British Journal of Ophthalmology 79.1 (1995): 777-786.

29. Stephen BK., et al. "Simplifying collection of corneal specimens in cases of suspected bacterial keratitis". Journal of Clinical Microbiology 41.7 (2003): 3192-3197.

30. Al- Shakarachi F. "Initial therapy for suppurative microbial keratitis in Iraq". British Journal of Ophthalmology 91.12 (2007): 1583-1587.

31. Bourcier T., et al. "Bacterial keratitis: predisposing factors, clinical and microbiological review of 300 cases". British Journal of Ophthalmology 87.1 (2003): 834-838.

32. Alexandrakis G., et al. "Shifting trend of bacterial keratitis in south Florida and emerging resistance to fluoroquinolones". Ophthalmology 107.1 (2000): 1497-1502.

33. Mozayemi RM and Lam S. "Phyctenular keraconjunctivitis and marginal Staphylococcal Keratitis". Indian Journal of Ophthalmology 1.1 (1996): 1392-1395.

34. Norina TJ., et al. "Microbial keratitis: aetiological diagnosis and clinical features in patient admitted to hospital university sains Malaysia". Singapore Medical Journal 49.1 (2008): 67-71.

35. Liesegang TJ and Forster RK. "Spectrum of microbial keratitis in South Florida". American Journal of Ophthalmology 90.1 (1980): 38-47.

36. Goldstein MH., et al. "Emerging fluoroquinolone resistance in bacterial keratitis: a 5-year review". Ophthalmology 106.1 (1999): 1313-1318.

37. Tiwari HK., et al. "Assessment of different tests to detect methicillin resistant Staphylococcus aureus (VRSA) from a tertiary care hospital in northern parts of India". BMC Infectious Diseases 6.1 (2009): 156.
38. Song JH., et al. "The Asian network for surveillance of resistant pathogens (ANSORP) study group". Antimicrobial Agents and Chemotherapy 48.12 (2004): 4926-4928.

39. Tiwari HK and Sen MR. "Emergence of vancomycin resistant Staphylococcus aureus (VRSA) from a tertiary care hospital in northern parts of India". BMC Infectious Diseases 6.1 (2006) 156.

40. Chen CJ and Starr CE. "Epidemiology of Gram-negative conjunctivitis in neonatal intensive care unit patients". American Journal of Ophthalmology 145.6 (2008): 966-970.

41. Sherman NE., et al. "Pseudomonas aeruginosa and a proteomic approach to bacterial pathogenesis". Disease Markers 17.4 (2001): 285-293.

42. Sanjana RK., et al. "Prevalence and antimicrobial susceptibility pattern of methicillin-resistant Staphylococcus aureus (MRSA) in CMS- teaching hospital: a preliminary report". Journal of $\mathrm{Ne}$ pal Medical Association 6.1 (2010): 1-6.

43. Paez PL., et al. "Impact of ciprofloxacin and chloramphenicol on the lipid bilayer of Staphylococcus aureus: Changes in membrane potential". BioMed Research International 10 (2013): 1155

44. Goldstein FW and Acar JF. "Epidemiology of quinolone resistance: Europe and North and South America". Drugs 49.2 (1995): 36-42.

45. Dutta GN., et al. "Inactivation of chloramphenicol by Staphylococcus aureus biotype C from humans and animals". Indian Journal of Medical Research 113.1 (2001): 11-13.

46. Hiramatsu K., et al. "Methicillin resistant Staphylococcus aureus clinical strains with reduced vancomycin susceptibility". Journal of Antimicrobial Chemotherapy 40.1 (1997): 135-136.

47. Centers for Disease Control and Prevention (CDC). "Staphylococcus aureus resistant to vancomycin United States". Morbidity and Mortality Weekly Report 51 (2002): 565-567.

48. Ujwol B., et al. "Status of inducible clindamycin resistance among macrolide resistant Staphylococcus aureus". African Journal of Microbiology Research 10.9 (2016): 280-284.

49. Venubabu T., et al. "Vancomycin resistance among methicillin resistant Staphylococcus aureus isolates from intensive care units of tertiary care hospitals in Hyderabad". Indian Journal of Medical Research 134.5 (2011): 704-708. 
50. Sueke H., et al. "Minimum inhibitory concentrations of standard and novel antimicrobials for isolates from bacterial keratitis". Investigative Ophthalmology and Visual Science 51.5 (2010): 2519-2524.

\section{Assets from publication with us}

- Prompt Acknowledgement after receiving the article

- Thorough Double blinded peer review

- Rapid Publication

- Issue of Publication Certificate

- High visibility of your Published work

Website: https://www.actascientific.com/

Submit Article: https://www.actascientific.com/submission.php Email us: editor@actascientific.com

Contact us: +919182824667 